\title{
Temperature Dependence of Highly Excited Exciton Polaritons in Semiconductor Microcavities
}

\author{
Tomoyuki Horikiri ${ }^{1,2,3,4}$, Yasuhiro Matsuo ${ }^{1,3}$, Yutaka Shikano ${ }^{5,6}$, Andreas Löffler ${ }^{7}$, \\ Sven Höfling ${ }^{1,7}$, Alfred Forchel ${ }^{7}$, and Yoshihisa Yamamoto ${ }^{1,2,3,4}$ \\ ${ }^{1}$ National Institute of Informatics, 2-1-2 Hitotsubashi, Chiyoda-ku, Tokyo 101-8430, \\ Japan \\ ${ }^{2}$ E. L. Ginzton Laboratory, Stanford University, Stanford, CA 94305, USA \\ ${ }^{3}$ The University of Tokyo, 7-3-1 Hongo, Bunkyo-ku, Tokyo 113-8656, Japan \\ ${ }^{4}$ Center for Emergent Matter Science, RIKEN, Wakoshi, Saitama 351-0198, Japan \\ ${ }^{5}$ Research Center of Integrative Molecular Systems (CIMoS), Institute for Molecular \\ Science, 38 Nishigo-Naka, Myodaiji, Okazaki 444-8585, Japan \\ ${ }^{6}$ Schmid College of Science and Technology, Chapman University, Orange, CA 92866, \\ $U S A$ \\ ${ }^{6}$ Technische Physik, Universität Würzburg, Am Hubland, D-97074 Würzburg, \\ Germany
}

Observations of polariton condensation in semiconductor microcavities suggest that polaritons can be exploited as a novel type of laser with low input-power requirements. The low-excitation regime is approximately equivalent to thermal equilibrium, and a higher excitation results in more dominant nonequilibrium features. Although standard photon lasing has been experimentally observed in the high excitation regime, e-h pair binding can still remain even in the high-excitation regime theoretically. Therefore, the photoluminescence with a different photon lasing mechanism is predicted to be different from that with a standard photon lasing. In this paper, we report the temperature dependence of the change in photoluminescence with the excitation density. The second threshold behavior transited to the standard photon lasing is not measured at a lowtemperature, high-excitation power regime. Our results suggest that there may still be an electron-hole pair at this regime to give a different photon lasing mechanism.

KEYWORDS: polariton, photon lasing, semiconductor, microcavity, Bose-Einstein condensation 


\section{Introduction}

Bosonic quasiparticles resulting from the strong coupling between photons and excitons are known as polaritons. Exciton polaritons are manifested by the spectroscopic observation of the photoluminescence (PL) from semiconductor microcavities. When the rate of inflow to the ground state of polaritons exceeds the rate of loss from the cavity, final-state stimulation can result in a macroscopic population of the ground state, and ground-state polariton condensation can occur. ${ }^{1-3}$ This defines the condensation threshold, which can be experimentally confirmed by a nonlinear increase in the PL from a semiconductor microcavity as a function of the input pump power, as polaritons inside the cavity cannot be observed directly. Owing to the short lifetime of polaritons, this condensation is treated as a nonequilibrium dynamical condensation; ${ }^{4}$ however, under certain conditions, such as polariton Bose-Einstein condensation (BEC), it may be treated as a thermal equilibrium condensation. ${ }^{5}$

There is a large body of experimental evidence that suggests that polariton condensation is a weakly interacting BEC induced by the polariton-polariton interaction. For example, observations of superfluidity, ${ }^{6}$ vortex formation, ${ }^{7}$ vortex-antivortex pair creation, ${ }^{8}$ and the spectrum of the Bogoliubov excitation ${ }^{9}$ all support this hypothesis. Furthermore, polariton condensation has the potential to be exploited for developing a novel type of laser with low input-power requirements. ${ }^{10}$ Unlike standard photon lasing in a semiconductor, population inversion is not needed, and an increase in the pump power leads to an increase in the strength of polariton-polariton interaction effects owing to the population increase. At very high densities, exciton overlap screens the Coulomb interaction, which breaks electron-hole $(\mathrm{e}-\mathrm{h})$ pairs and results in an $\mathrm{e}-\mathrm{h}$ plasma system. Population inversion then occurs, and photon lasing is observed after the second nonlinear increase, which is often called the second threshold in contrast to the first or condensation threshold. ${ }^{11-18}$ However, recent theoretical studies ${ }^{19-23}$ have indicated that some $\mathrm{e}-\mathrm{h}$ pairs can remain in the high-density regime.

The aim of this study is to clarify whether the high excitation regime can be detected in the PL behavior, as direct observation of $\mathrm{e}-\mathrm{h}$ pairs and the $\mathrm{e}-\mathrm{h}$ plasma is not possible. To compare the PL behavior in the high-excitation regime with standard photon lasing, we investigated the temperature dependence of the PL because the high-temperature case shows standard photon lasing. Here, we demonstrate a completely different PL behavior from that of standard photon lasing at a low temperature $(8 \mathrm{~K})$ in the high- 
(a) Zero detuning (strong coupling)

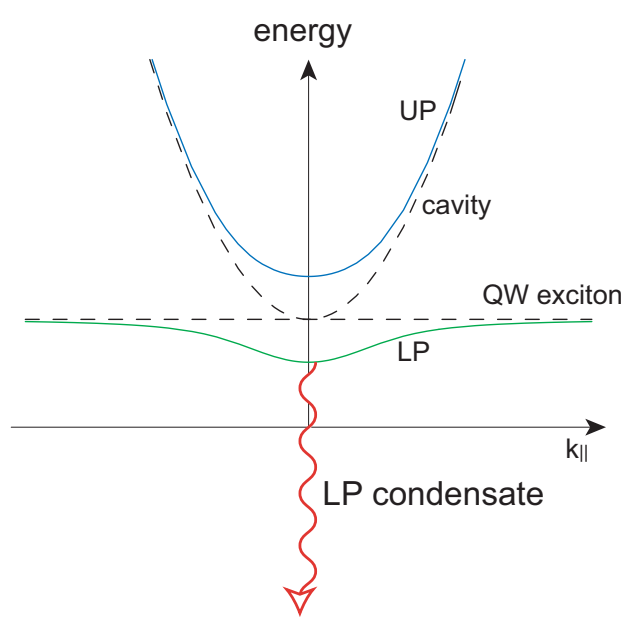

(b) Large detuning or high temperature (weak coupling)

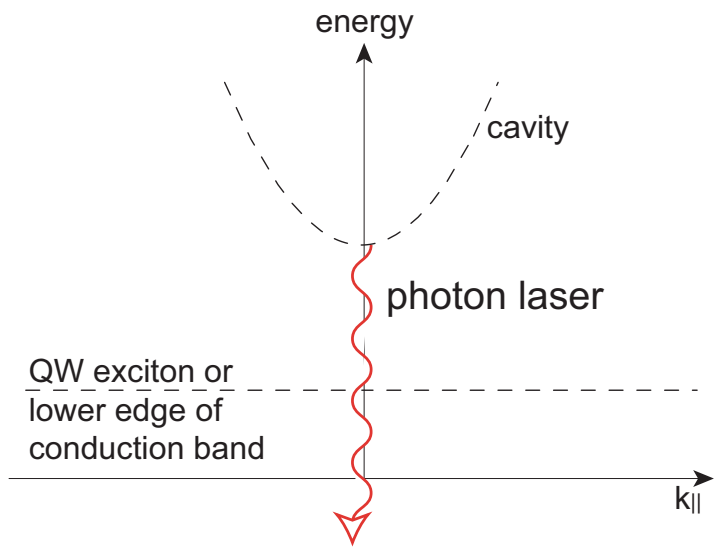

Fig. 1. (Color Online) Energy-momentum relationships for the strong and weak coupling regimes. (a) Polariton condensation. (b) Photon lasing, for which population inversion is necessary.

excitation regime.

In $\S 2$, we present the basic concepts of semiconductor microcavity polaritons and review recent developments in the high-density regime in $\S 3$. In $\S 4$, the results of the excitation dependence measurements, in which the PL intensity, energy, PL linewidth, and second-order correlation function are studied as functions of pump power, are presented. These results are discussed with regard to temperature to compare the condensation and lasing mechanisms in $\S 5$. Furthermore, we evaluate the excitation density of the $\mathrm{e}-\mathrm{h}$ pairs in our experimental setup to identify the high-excitation regime for which the PL behavior is different from standard photon lasing. Section 6 is devoted to a summary.

\section{Polariton Condensation and Photon Lasing}

Figure 1 (a) shows that, in a semiconductor microcavity with a quantum well (QW), a polariton is created by the strong coupling between the QW exciton and a cavity photon, with the exciton itself formed by e-h binding. As a result of fast energy transfer between two cavity modes, normal-mode splitting occurs, and two energy branches referred to as the upper polariton (UP) and lower polariton (LP) are formed. When the conditions in the cavity meet those of the condensation threshold, a polariton condensate is formed in the LP ground state, that is, when the rate of injection into the LP ground state through relaxation by $\mathrm{e}-\mathrm{h}$ pair, $\mathrm{e}-\mathrm{h}$ and phonon, polariton-polariton, and 
polariton-phonon scattering is higher than the rate of loss from the ground state owing to inverse processes and cavity leakage. The macroscopic population in the LP ground state then grows, and a nonlinear increase in the PL is observed. This condensate is often called the polariton laser. The difference between this and photon lasing is that the strong PL emission is not caused by a conventional lasing mechanism, i.e., light amplification by stimulated emission of radiation.

Conventional semiconductor photon lasing in a photon laser, as shown in Fig. 1 (b), occurs when there is no longer any strong coupling between the cavity photon and the QW exciton. At room temperature, excitons dissociate owing to the high thermal energy, and since the exciton level no longer exists in a standard semiconductor, such as GaAs, the cavity energy in the conduction band is that of an e-h plasma. Photon lasing relies on population inversion with respect to the ground state, but there are also other differences between polariton condensation and photon lasing.

The normalized second-order correlation function $g^{(2)}(0)$ of photon lasing converges to unity just above the lasing threshold, ${ }^{24}$ while that of the polariton condensate shows bunching $\left(g^{(2)}(0)>1\right)$ and super-Poisson statistics above the condensation threshold. ${ }^{4,25}$ This can be understood as being a result of the interactions between condensate polaritons or between a polariton and phonon, which deplete the condensate population as polaritons are lost from the cavity as a result of these interactions. ${ }^{3,26-29}$ Owing to this mechanism, higher-order coherence of the condensate is not realized, and in fact, the third-order correlation function $g^{(3)}(0)$ has been shown to be larger $\left(g^{(3)}(0)>g^{(2)}(0)>1\right) .{ }^{30}$ The coherent state exhibits the all-order coherence $g^{(n)}(0)=1$, while the thermal state exhibits $g^{(3)}(0)=6>g^{(2)}(0)=2>1$. Consequently, the statistics of the PL from the polariton condensate indicate that the condensate is not in the coherent state but close to the thermal distribution state.

Table I. Features of polariton condensation and photon lasing. The cavity photon energy is $\omega_{\text {cav }}$, $\omega_{\mathrm{LP}}$ is the LP ground state energy, and $\mu$ is the condensate blue shift due to the interactions.

\begin{tabular}{cccc}
\hline & $g^{(2)}(0)$ & Energy level & Linewidth \\
\hline Polariton condensate & $>1$ & $\omega_{\mathrm{LP}}+\mu$ & Broadening owing to p-p interaction \\
Photon laser & $\rightarrow 1$ & $\omega_{\text {cav }}$ & Narrowing owing to cavity linewidth \\
\hline
\end{tabular}

The differences between polariton condensation and photon lasing are summarized in Table I. Differences in the energy and linewidths, which are observed in the PL 
measurements, also arise. In polariton condensation, the PL energy differs from that of the LP ground state $\left(\omega_{\mathrm{LP}}\right)$ by a small blue shift due to polariton interactions $(\mu)$, while the energy of the photon laser is the same as the cavity photon energy of zero in-plane momentum $\left(\omega_{\text {cav }}\right)$. The polariton linewidth broadens as the density increases owing to the polariton-polariton $(\mathrm{p}-\mathrm{p})$ interactions,${ }^{31}$ while the photon laser shows a narrow linewidth owing to their absence.

\section{Behavior in a High Excitation Regime}

When a polariton system reaches the condensation regime above the condensation threshold, a transition to photon lasing in a higher-excitation regime is considered to occur. ${ }^{11-18,32}$ The PL intensity has a second threshold, ${ }^{11,15,32}$ where population inversion is believed to be the reason for the transition to photon lasing. In ref. 16, a study of the Bernard-Duraffourg condition, which defines photon lasing conditions in semiconductors, revealed that the system was in a regime close to the inversion threshold at the onset of photon lasing. The observation of the second-order coherence function $g^{(2)}(0)$ has interestingly shown that it monotonically converges to unity ${ }^{17,18}$ above the photon lasing threshold. The results of these studies can be understood as follows. With an increase in the population, $\mathrm{e}-\mathrm{h}$ binding in the high excitation regime weakens owing to the screening effect between $\mathrm{e}-\mathrm{h}$ pairs, and the excitons become an $\mathrm{e}-\mathrm{h}$ plasma. Consequently, standard photon lasing takes place after the gain for the cavity photon mode exceeds its loss. However, it is unclear whether the breaking of $\mathrm{e}-\mathrm{h}$ pairs, determined by the $\mathrm{e}-\mathrm{h}$ interaction and $\mathrm{e}-\mathrm{h}$ density, necessarily occurs in every microcavity $\mathrm{QW}$ sample. The polariton condensate is expected to experience several phases governed by the excitation density and detuning, for which different signatures of the photon laser have been theoretically determined under suitable conditions. ${ }^{19-23}$ In particular, the work described in refs. 22 and 23 utilized the framework of a nonequilibrium system caused by pumping and decay, ${ }^{33}$ which is very close to the actual experimental conditions. In the high-density regime, $\mathrm{e}-\mathrm{h}$ binding is no longer a result of the Coulomb interaction; instead, photon induced attraction takes place. As a result, a unique dip in the momentum distribution (kinetic hole burning) appears. ${ }^{22}$

In these studies, in which a transition to photon lasing in the high-density regime was observed, polaritons no longer survived after the transition; excitons dissociated and formed an e-h plasma in the high-excitation regime. However, theoretical studies predict that another regime that does not necessarily experience $\mathrm{e}-\mathrm{h}$ pair breaking should exist 
in the high-density regime. There are currently no experimental conditions that are known to implement $\mathrm{e}-\mathrm{h}$ pair binding at high densities; however, by exploring different Q-factors, detuning values, excitation properties (angle, spot size, and energy), and a lower heating effect from the pumping laser, it may be possible to observe differences in the PL behavior from those of photon lasing. In several studies, the LP resonance has been utilized, but there is some disagreement over whether the coherence from the pumping laser can be transferred to the polariton condensate since the energy is close to the pumping energy and populated within a few scattering events. Therefore, the pumping energy (UP or cavity resonance at a large momentum) was chosen for this work in such a way that a high input rate and a high excitation density were obtained. To do so, the angle needs to be tuned to the reflection dip of the sample. To guarantee a sufficiently high energy, the reflection dip of the stop band edge of a distributed Bragg reflector (DBR) structure was used because we were not able to operate the laser stably at high energies. In terms of the spot size, if normal pumping was performed, then a clearer, smaller pumping for the condensate spatial profile might be obtained, but this was not possible owing to the limited pumping energy of the sample. Previous studies have considered samples with different Q-factors, but the unique behavior we report here was only obtained in the DBR sample we used. Thus, in what follows, we will examine the trends in the PL behavior on the basis of the temperature and excitation density of the sample. We find that a transition to photon lasing does not completely explain the results at low temperatures, which imply that the effects of $\mathrm{e}-\mathrm{h}$ pairs are still present in the high-excitation regime.

\section{Temperature and Excitation Power Dependences of PL}

The low-temperature condition in which a polariton condensate can be formed may show a different behavior from that of a photon laser, and to compare the results of the temperature and excitation density dependences of the $\mathrm{PL}^{18}$ with the photon lasing case, we also present high-temperature measurements for which $\mathrm{e}-\mathrm{h}$ pairs dissociate owing to the thermal energy and the cavity photon mode is in the conduction band. Moreover, additional experiments at intermediate temperatures also show an interesting behavior, including the appearance of the second threshold of the transition from a polariton condensate to a photon laser, which highlights the absence of the second threshold of the PL intensity at low temperatures. 


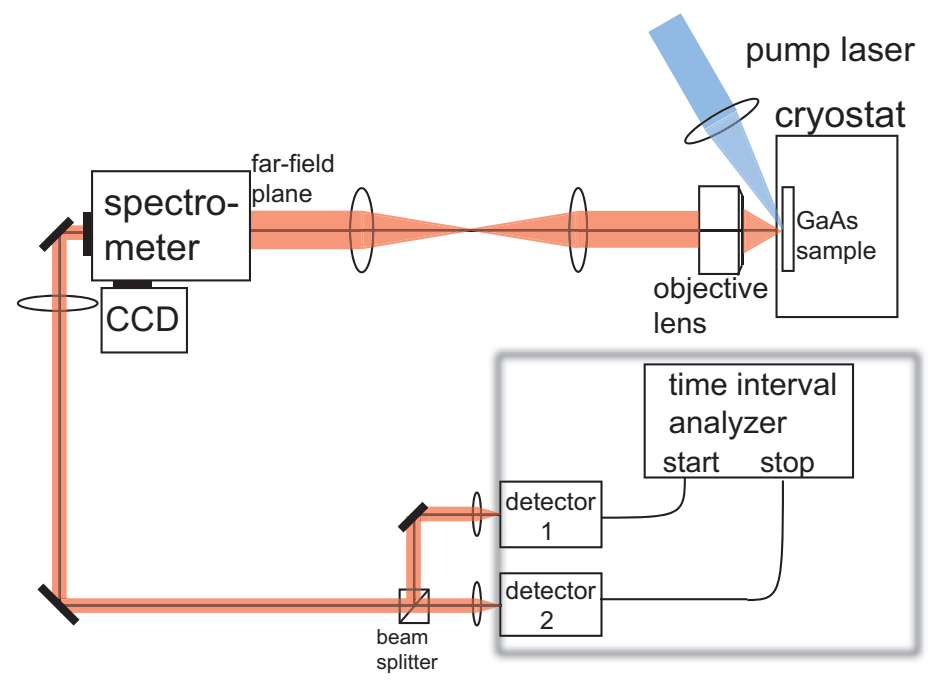

Fig. 2. (Color Online) Experimental setup for the dispersion relation measurements. The blue beam is that of the pump laser, while the red beam represents the PL from the sample. For time-resolved spectroscopy, the spectrometer was replaced by a streak camera attached to a monochromator.

\subsection{Experimental setup}

The sample had a microcavity structure consisting of AlAs/AlGaAs DBRs. To obtain the PL from the top surface, DBRs with 16 (20) top (bottom) layers were used. The 12 QWs were divided into three groups and positioned at the three highest-modeintensity antinodes of the microcavity. This sample showed strong coupling at low temperatures, and the normal-mode splitting at $k=0$ was $14 \mathrm{meV}$ around zero detuning. The pump laser was a mode-locked Ti:sapphire laser with a 3 ps pulse width; light was injected into the sample at an angle of 50-60 from the normal, which corresponds to $k_{\|} \sim 7 \times 10^{4} \mathrm{~cm}^{-1}$. The wavelength of the pump laser was set to $747 \mathrm{~nm}$ at $8 \mathrm{~K}$ to maximize the rate of injection into the sample reflection dip due to the cavity photon mode. As the temperature was increased, the cavity photon energy experienced a redshift, which meant that the pump-laser wavelength needed to be changed with temperature.

A schematic of the experimental setup is shown in Fig. 2. A total of three lenses including an objective lens with a large numerical aperture of 0.55 for large $\Delta k$ collection were used for imaging the far-field plane on the entrance slit of the spectrometer. The imaging of the far-field plane means that the radial coordinate corresponds to the PL emission angle from the sample, with the in-plane momentum of the polariton or the photon in the sample being defined as $k_{\|}=2 \pi / \lambda \sin \theta$, where $\lambda$ is the PL wavelength and $\theta$ is the PL emission angle. The emission in the horizontal direction is deflected in 
the spectrometer by a grating to create the energy axis. Since the vertical axis corresponds to the in-plane momentum, the dispersion relation is recorded by a CCD camera attached to one exit of the spectrometer. The other exit of the spectrometer is used to send the PL to a Hanbury Brown and Twiss (HBT) setup to measure the secondorder correlation function. The HBT setup consists of a beam splitter, single-photon detectors (Perkin Elmer, SPCM-AQRH-14), and a time-interval analyzer (ORTEC, picosecond time analyzer 9308). The spectrometer grating is adjusted after every measurement at each temperature to obtain the whole spectrum since the PL energy shifts as the excitation density changes when the pump laser power changes. The width of the spectrometer exit slit, corresponding to the width of the observed energy, is set so as to obtain a sufficiently wide spectrum with the HBT setup ( $\sim \mathrm{nm})$. The detection width of the in-plane momentum is $\Delta k \sim 2 \times 10^{4} \mathrm{~cm}^{-1}$. When we perform time-resolved spectroscopy, the spectrometer is replaced by a streak camera with a 2 ps time resolution. However, the time resolution is degraded by the temporal dispersion of the grating inside the monochromator attached to the front of the streak camera.

\subsection{Temperature dependence below threshold}

Table II. Effective masses and detuning values at various temperatures. The effective mass of the cavity was evaluated to be $m_{\text {cav }}=2.8 \times 10^{-35} \mathrm{~kg}$.

\begin{tabular}{cccccc}
\hline Temperature $(\mathrm{K})$ & 8 & 50 & 70 & 100 & 200 \\
Effective mass $\left(m_{\text {cav }}^{-1}\right)$ & 2.5 & 2.8 & 2.9 & 4.4 & 1 \\
Detuning $(\mathrm{meV})$ & 2.5 & 3.8 & 4.5 & 9.0 & - \\
\hline
\end{tabular}

We began with an investigation of the temperature dependence of the normal-mode dispersion relations seen in the low-excitation regime below the condensation or lasing threshold. As the temperature increased, we found that both the exciton energy and the cavity photon energy were redshifted. The redshift of the exciton energy occurred faster than that of the cavity photon energy. Furthermore, the detuning $\Delta E\left(k_{\|}\right)=E_{\text {cav }}\left(k_{\|}\right)-$ $E_{\text {exc }}\left(k_{\|}\right)$, where $E_{\text {cav }}\left(k_{\|}\right)$is the cavity photon energy and $E_{\text {exc }}\left(k_{\|}\right)$is the exciton energy, increased as the system temperature increased. We experimentally measured dispersion curves, fitted a quadratic curve to the dispersion curve near $k_{\|}=0$, and estimated the effective mass from the curvature. In the case of the LP branch, a wider fitting region resulted in a heavier evaluated effective mass (see Fig. A.1 in Appendix A for the fitting 
(a) $8 \mathrm{~K}$

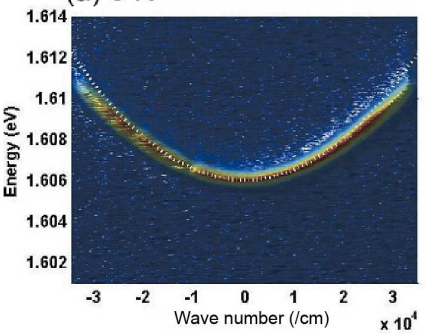

(d) $100 \mathrm{~K}$ (b) $50 \mathrm{~K}$

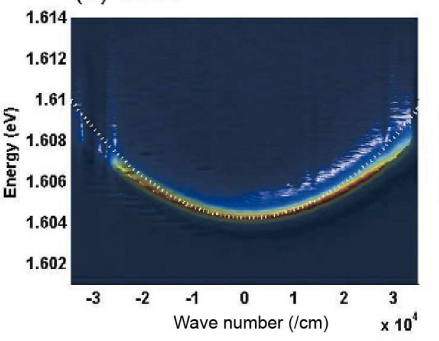

(c) $70 \mathrm{~K}$

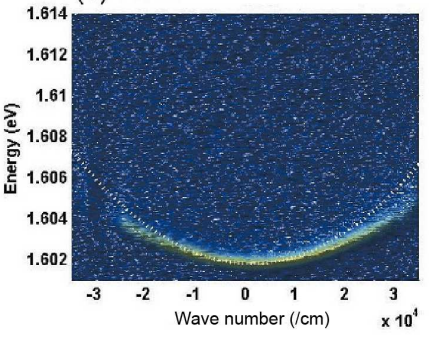

(e) $200 \mathrm{~K}$
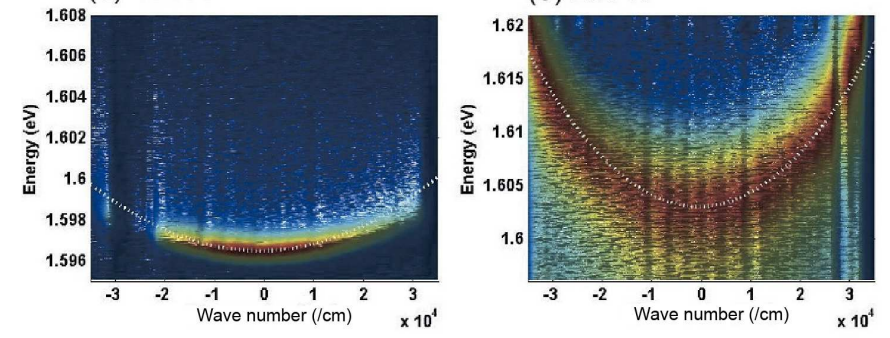

Fig. 3. (Color Online) Dispersion relations below threshold at (a) 8, (b) 50, (c) 70, and (d) $100 \mathrm{~K}$. The dispersion for the LP branch is shown. Cavity-mode branch dispersion was observed at (e) $200 \mathrm{~K}$. As the temperature increased, the effective mass became heavier and the detuning increased because the energy of the exciton was redshifted as the temperature of the exciton increased faster than the cavity temperature. At $200 \mathrm{~K}$, the effective mass was the same as the mass of the cavity photon since strong coupling no longer existed owing to the large detuning, and the system was in the weak-coupling regime.

region).

Dispersions taken below the threshold at various temperatures obtained by timeintegrated far-field spectroscopy are shown in Fig. 3. These dispersions represent the temperature-induced transition from the small-mass LP mode at $8 \mathrm{~K}$ to heavier excitonlike heavier LP modes at higher temperatures of 50, 70, and $100 \mathrm{~K}$, and then the further transition to the cavity mode at $200 \mathrm{~K}$ (the lasing threshold is reached in this mode). Note that the lower branch changed from that of the LP to that of the exciton, and so no threshold behavior was seen in the lower energy branch at $200 \mathrm{~K}$.

The detuning values, listed in Table II along with the effective masses, were evaluated from the cavity photon mass at $200 \mathrm{~K}$ and the effective mass of the LP. The refractive index of 3.14 for the cavity photon mass at $200 \mathrm{~K}$ was lower than that of GaAs because of the layered DBR structure containing $\mathrm{Ga}_{0.8} \mathrm{Al}_{0.2} \mathrm{As}$ and $\mathrm{AlAs}$, whose refractive indexes are smaller than that of GaAs. At $8 \mathrm{~K}$, the LPs relaxed to the bottom of the branch and formed a polariton condensate when the pump power was increased. As the temperature 
increased, the effective mass increased, as did the detuning. The LP mode became exciton-like owing to the fast redshift of the exciton energy, before separating into heavy hole (hh) exciton and cavity photon modes, which is accompanied by a transition from strong coupling to weak coupling. At $200 \mathrm{~K}$, the smaller effective mass indicates the cavity photon mode.

This study differs from that in ref. 18 is that the sample position was not changed as the detuning was scanned in response to the temperature change. This allowed us to screen out unexpected effects due to position-dependent imperfections in the sample and was done partly because the temperature dependence of constant detuning has already been studied in ref. 18. Since our aim was to investigate standard vertical cavity surface-emitting laser (VCSEL)-type lasing, where there is no strong coupling between an exciton and a cavity photon below the lasing threshold at $200 \mathrm{~K}$, this high-temperature condition at which exciton binding is difficult was suitable.

\subsection{Excitation power dependence}

In examining the dependence of the normal modes on the excitation density at several temperatures, we found that the PL of the high-density polariton condensates at a low temperature $(8 \mathrm{~K})$ displayed distinct features in terms of the PL intensity, energy, linewidth, and second-order correlation function compared with the PL of the photon laser obtained at a high temperature (200 K). Moreover, the temperature data ranging from 8 to $200 \mathrm{~K}$ clearly shows how each feature of the polariton condensate converges to each of those of the photon laser.

\subsubsection{PL intensity}

The excitation power dependence of the PL intensity, shown as a function of the pump laser power, for various temperatures is shown in Fig. 4. A nonlinear increase in the PL intensity is seen for each temperature, and as the temperature was increased, the threshold laser power, i.e., the threshold excitation density, increased. A higher excitation density is needed to reach the lasing threshold and therefore achieve population inversion, whereas this is not necessary for the polariton system. ${ }^{10}$ Note that the normal modes below the threshold for temperatures lower than $100 \mathrm{~K}$ were the LP and UP, and we can see that the LP branch reached the threshold. By increasing the temperature, the UP gradually changes to the cavity mode, and LPs become excitons with the flat curvature of a heavy effective mass. Thus, the normal mode at $200 \mathrm{~K}$ was the cavity 


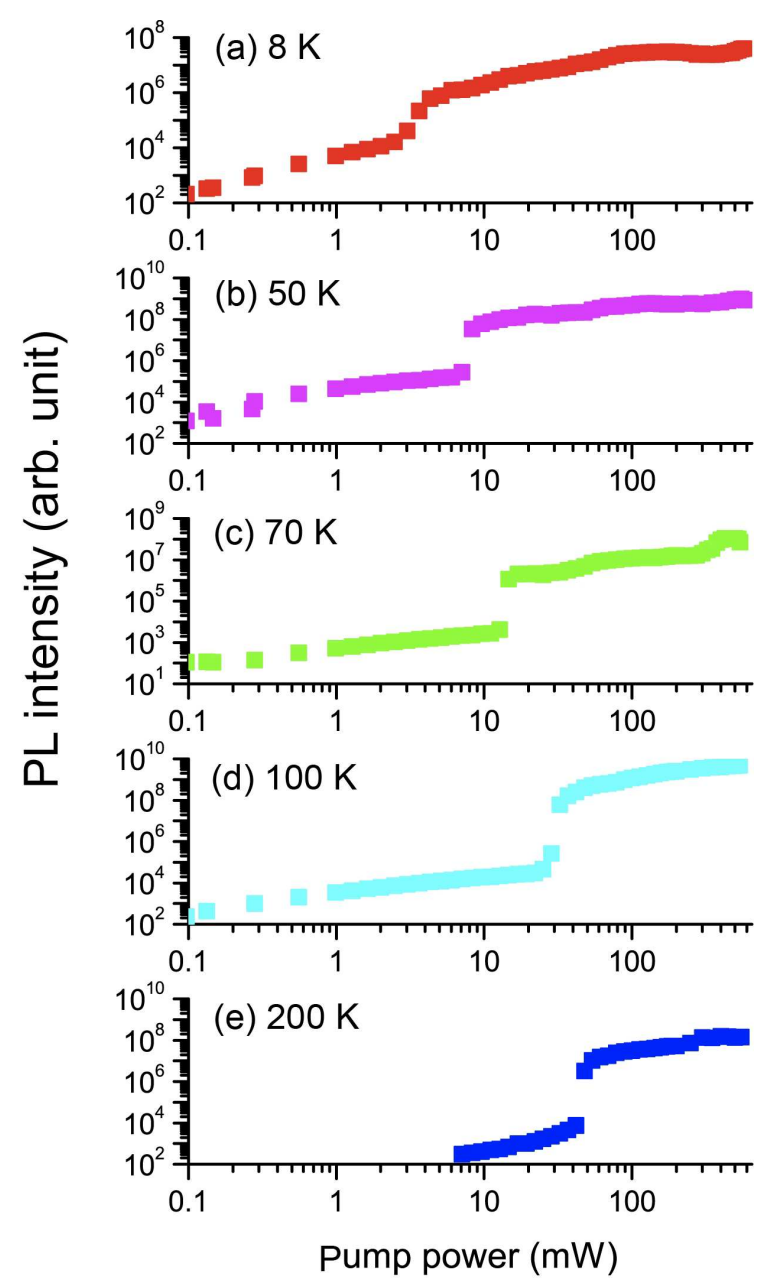

Fig. 4. (Color Online) Excitation power dependence of the PL intensity at $k=0$. (a) 8, (b) 50, (c) 70, (d) 100, and (e) $200 \mathrm{~K}$. At $200 \mathrm{~K}$, the PL intensity was weak and undetectable below $6 \mathrm{~mW}$.

mode due to exciton dissociation and the large detuning, that is, the cavity mode was already in the conduction band. The transition of the cavity mode to the lasing mode occurred at $200 \mathrm{~K}$.

The lowest threshold pump power of approximately $3 \mathrm{~mW}$ was recorded for the 8 $\mathrm{K}$ case [Fig. 4 (a)], and as the excitation increased, a monotonic increase in the PL intensity was seen. However, a distinct second threshold was not observed, unlike in previous studies. ${ }^{11-17}$ As expected from BEC thermal equilibrium theory, the threshold increased with an increase in the temperature but the second threshold was not observed until $70 \mathrm{~K}$ [Fig. 4 (c)], where a nonlinear PL intensity increase was seen at around 300 $\mathrm{mW}$, implying a transition to photon lasing. At $100 \mathrm{~K}$ and $200 \mathrm{~K}$ [Figs. 4 (d) and (e)], the second threshold was not observed, which implies that the system has already reached photon lasing after the first threshold. However, as we will show later, the 
properties of the second-order correlation function make this slightly ambiguous in the case of $100 \mathrm{~K}$.

\subsubsection{PL energy}

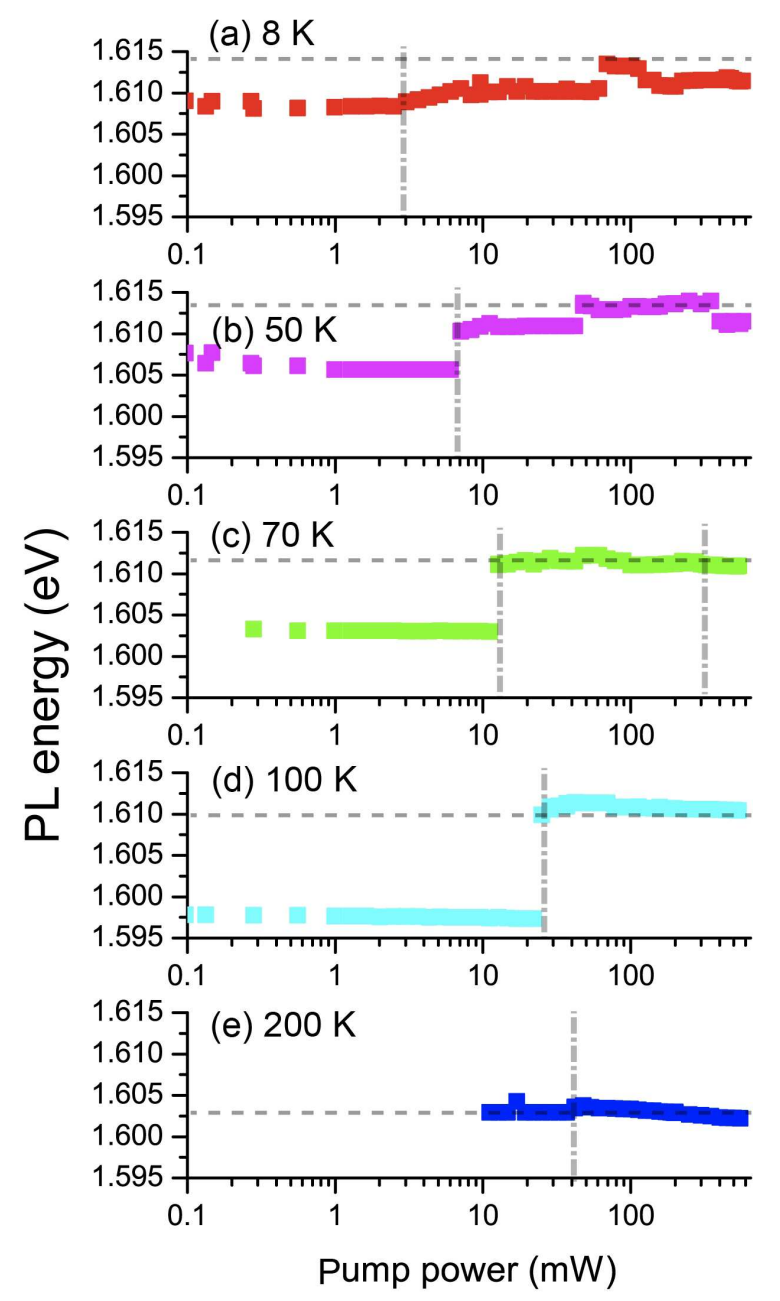

Fig. 5. (Color Online) Excitation power dependence of the PL energy. The dashed lines are the energy of a cavity photon of $k=0$ at each temperature. The discrepancy between the dashed line and the PL energy at $100 \mathrm{~K}$ is due to the low precision in evaluating the cavity energy from the effective mass. The limitation in the precision comes from the broad linewidth due to the thermal energy. The dot-dashed lines correspond to the thresholds in the PL intensity.

Looking at the change in the PL energy at $k=0$, which gives the maximum $\mathrm{PL}$ intensity at each temperature (Fig. 5), we see that in the $8 \mathrm{~K}$ case [Fig. 5 (a)] there is a blue shift in the energy below the condensation threshold $(\sim 1.608 \mathrm{eV})$ at approximately the condensation threshold $(\sim 3 \mathrm{~mW})$. The energy gradually approached the cavity photon energy of $1.615 \mathrm{eV}$ as the pump power increased. The behavior was also seen 


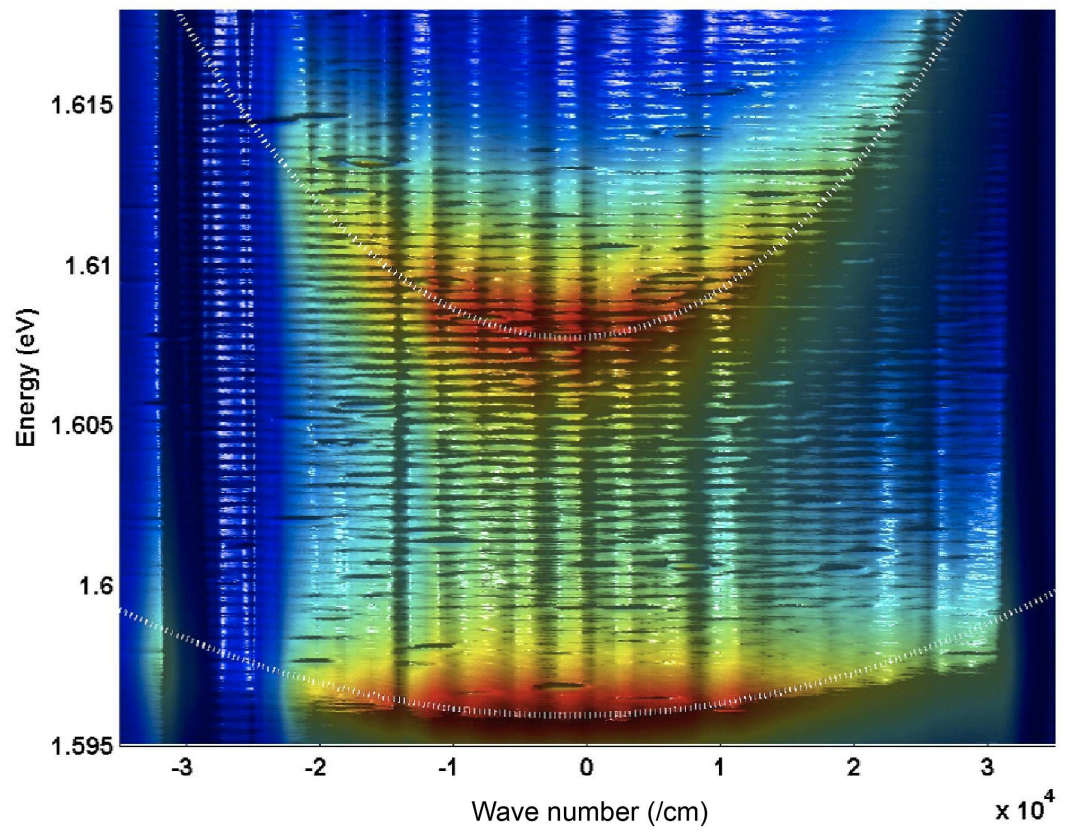

Fig. 6. (Color Online) Dispersion around the threshold at $100 \mathrm{~K}$.

at $50 \mathrm{~K}$ [Fig. $5(\mathrm{~b})$ ], where the condensation threshold was around $6 \mathrm{~mW}$. Note that the increase in temperature resulted in a redshift of the energy below the threshold, but a large blue shift of $5 \mathrm{meV}$ occurred at the threshold. This blue shift was much larger than that in the $8 \mathrm{~K}$ case because the large excitonic component at $50 \mathrm{~K}$ resulted in a large interaction energy. With further increase in the excitation density, there is another jump in the PL energy at around $40 \mathrm{~mW}$ to an energy $7 \mathrm{meV}$ higher than that below the condensation threshold and equal to the cavity photon energy. However, even under these conditions, the lower-energy peak (5 meV higher than the energy below the threshold) reappeared in the higher-excitation regime at around $400 \mathrm{~mW}$; a similar behavior was observed in the data at $8 \mathrm{~K}$. Owing to the pumping of the pulse laser, the polariton density showed a pulsed profile on a time scale of 1-10 ps. The PL emitted at the maximum excitation density originates from the cavity phonon energy, and as the density decreased, the energy of the PL also decreased.

A different behavior was observed at $70 \mathrm{~K}$ [Fig. 5 (c)]. A large blue shift of $7 \mathrm{meV}$ was seen at the first threshold at around $10 \mathrm{~mW}$, but there was no large change in the energy in the high-excitation regime, which may be due to heating by the strong pump laser. The system remained in the weak-coupling regime even when the excitation density decreased with a pulsed excitation profile. At the second threshold $(\sim 300 \mathrm{~mW})$, 
the energy corresponding to the maximum PL intensity remained the same as the cavity photon energy. However, the presence of this second threshold raises the possibility that the behavior in the intermediate regime differs from photon lasing.

The $100 \mathrm{~K}$ case [Fig. 5 (d)] showed a similar behavior to the $70 \mathrm{~K}$ case, except for the amount of blue shift at the first threshold. At low temperatures, the normal-mode splitting was $14 \mathrm{meV}$, and the cavity photon energy was midway between the UP and the LP. However, the detuning at $100 \mathrm{~K}$ was large, and the cavity photon energy was very close to the UP energy. Therefore, the blue shift was larger than those at lower temperatures and almost the same as that of normal mode splitting. In Fig. 6, the curvature of the lower energy mode reveals a heavy LP mass due to the large detuning at $100 \mathrm{~K}$, while the upper energy mode shows the curvature of the cavity photon mode, which is brighter around the threshold pump power. The energy above the threshold is equal to the cavity photon energy, implying photon lasing.

\subsubsection{PL linewidth}

Turning to the linewidth, narrowing at the threshold was observed in the $8 \mathrm{~K}$ case [Fig. 7 (a)], but this was followed by broadening as the density increased owing to both polariton-polariton interactions ${ }^{31}$ and pulsed excitation since the amount of blue shift depends on the excitation density and the PL from various densities was integrated at the CCD. The gradual narrowing of the linewidth in the high-density regime shows that there are a possible concentration of the cavity photon energy and a decrease in polariton-polariton interaction, which indicate an approach to photon lasing. This trend was repeated at $50 \mathrm{~K}$ [Fig. 7 (b)], but when the maximum PL energy was equal to the cavity photon energy at around $40 \mathrm{~mW}$, no further broadening occurred, with the linewidth being around $4 \mathrm{meV}$, as in the $8 \mathrm{~K}$ case. The broad linewidth is a result of the PL from not only the cavity photon energy but also the lower energy, as discussed in the previous subsection.

Although linewidth narrowing at the threshold and the subsequent broadening was also observed at $70 \mathrm{~K}$ [Fig. 7 (c)], the maximum linewidth was less than $3 \mathrm{meV}$. The narrower linewidth is due to the fact that there was only one peak at the cavity photon energy. However, this linewidth was still larger than that in the photon lasing case [Fig. 7 (e)] and implies that a polariton-polariton interaction effect remains. At the second threshold $(300 \mathrm{~mW})$, narrowing due to the PL concentrating to the cavity photon energy was observed, and a clear transition to photon lasing occurred. At $100 \mathrm{~K}$ [Fig. 7 


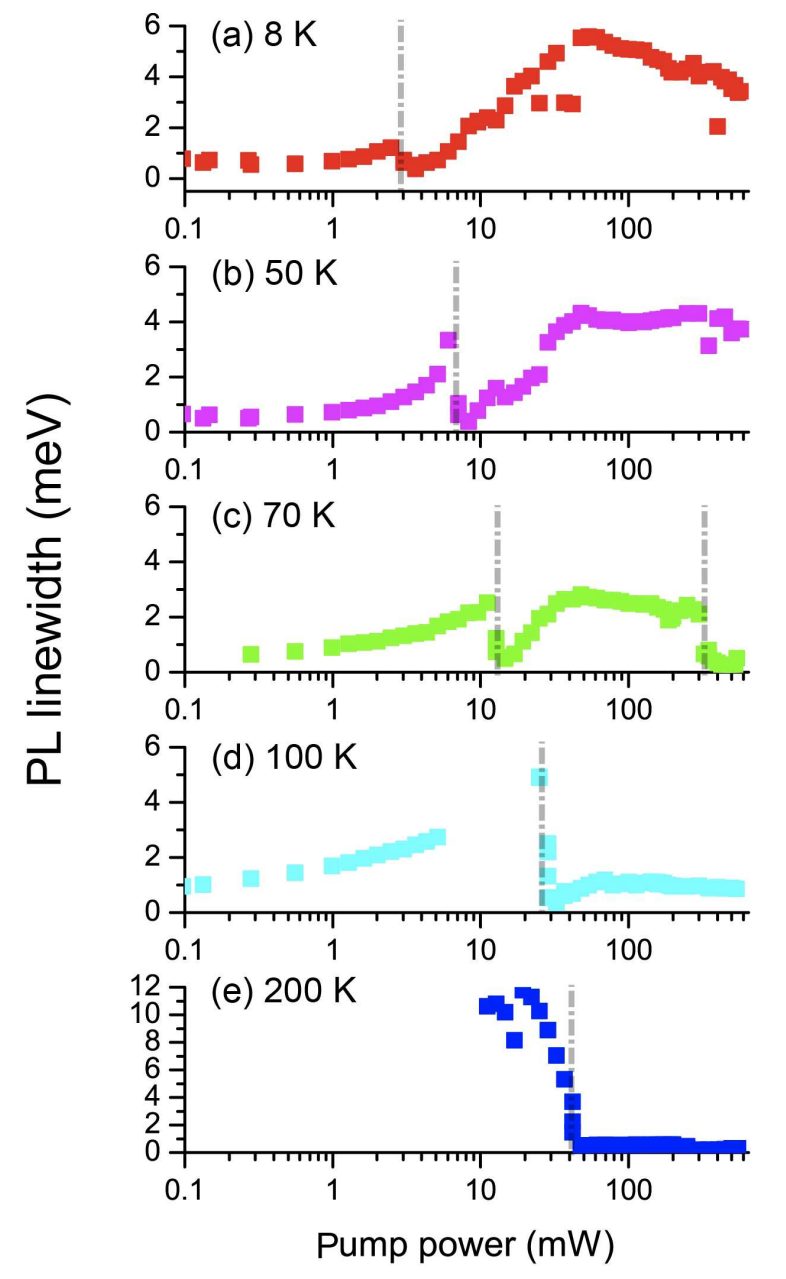

Fig. 7. (Color Online) Excitation power dependence of the PL linewidth. The dot-dashed lines correspond to the thresholds in the PL intensity.

(d)], narrowing occurred at the threshold; however, the subsequent broadening was smaller than that at $70 \mathrm{~K}$ because of a decrease in the polariton-polariton interaction effect. At $200 \mathrm{~K}$ [Fig. 7 (e)], the broad linewidth below the threshold caused by the high thermal energy narrowed drastically at the lasing threshold. The PL energy in this case was equal to the cavity photon energy since there was no polariton-polariton interaction effect, and hence, no linewidth broadening occurred above the lasing threshold.

Although a narrow linewidth was observed at temperatures above $70 \mathrm{~K}$, in the lowtemperature cases ( 8 and $50 \mathrm{~K}$ ), the broad linewidth in the higher excitation regime implies that polariton-polariton interactions maintain their effect even in a high-density regime on the order of a hundred times the condensation threshold. 


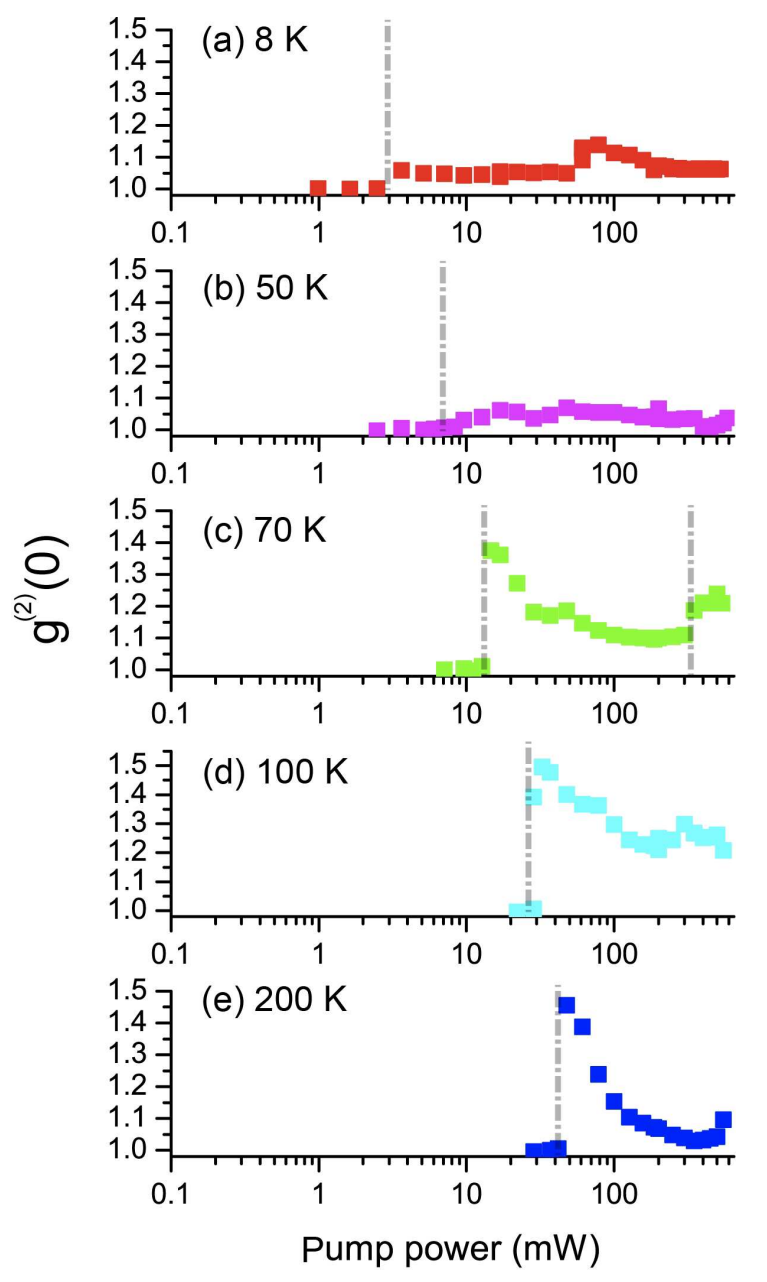

Fig. 8. (Color Online) Excitation power dependence of the second-order coherence function. The dot-dashed lines correspond to the thresholds in the PL intensity.

\subsubsection{Second-order correlation function}

We also examined the second-order correlation function (Fig. 8) using the HBT setup; however, difficulties in detecting weak signals prevented us from collecting data at low pump powers. In the $8 \mathrm{~K}$ case [Fig. 8 (a)], the PL showed bunching behavior above the threshold $\left(g^{(2)}(0)>1\right)^{4,17,18,25,30}$ up until the high-excitation-density regime, which was more than a hundred times the condensation threshold $(>300 \mathrm{~mW})$. When the measurements were performed using a high-time-resolution streak camera, ${ }^{17}$ the $g^{(2)}(0)$ below the threshold was closer to 2 after the statistics of the thermal state were correctly obtained. Note that, in this study, the time resolution of the photon detectors in the HBT setup ( $300 \mathrm{ps})$ was not sufficient to resolve the signal in one pulse. Average statistics were obtained because the intensity correlation time may be much shorter than the PL lifetime: we did not observe any correlation between the photons 
at the beginning and end of the PL pulse. ${ }^{4}$ The results for $g^{(2)}(0)$ were thus equal to unity.

When the system is in the condensation regime, the PL pulse width is reduced by more than one order of magnitude. ${ }^{17,18,30}$ The intensity correlation time of the system covers or is closer to the entire PL pulse. Therefore, even though the time resolution of the detectors is greater than the PL lifetime, the detection system correctly collects the photon statistics. ${ }^{25,30}$ If the actual correlation time is shorter than the pulse duration, then the $g^{(2)}(0)$ obtained underestimates its true value, ${ }^{25}$ and in fact, even if the measured results do not reflect the true statistics, they still indicate the bunching and the difference from photon lasing that occurs far above the threshold (see Appendix B for more details).

Bunching in the broad excitation density regime was also seen at $50 \mathrm{~K}$ [Fig. 8 (b)], but the strong bunching above the threshold at $70 \mathrm{~K}$ [Fig. 8 (c)] was different from that at low temperatures. The behavior at these two temperatures was similar to that in the case of photon lasing. $g^{(2)}(0)$ did not reach unity but rather increased after the second threshold, as also observed in a previous study. ${ }^{17}$ At $100 \mathrm{~K}$ [Fig. 8 (d)], the absence of a clear second threshold in the PL intensity meant that there was also no clear second threshold in $g^{(2)}(0)$; however a clear convergence to unity was not seen at this temperature, implying a difference from photon lasing although an even higher density might cause convergence. In the $200 \mathrm{~K}$ case [Fig. 8 (e)], $g^{(2)}(0)$ monotonically decreased to unity after the transition at the threshold, which is a typical characteristic of photon lasing. ${ }^{24}$

\section{Discussion}

In the previous section, we have reported several PL characteristics at various temperatures. In this section, we discuss the lasing mechanism at each the temperature.

At $200 \mathrm{~K}$, there are no $\mathrm{e}-\mathrm{h}$ correlations from the observation of the dispersion curve below threshold [Fig. 3 (e)]. The e-h plasma is formed, increasing the excitation power. Owing to the same microcavity structure as the VCSEL, conventional photon lasing occurs from the observations of the same energy of the PL peak to the cavity energy [Fig. 5 (e)], the narrow linewidth [Fig. 7 (e)], and the converged behavior of the second-order autocorrelation function [Fig. 8 (e)] above the first threshold. While the phenomena observed at $200 \mathrm{~K}$ is similar to those observed at $100 \mathrm{~K}$, particularly, the narrow PL linewidth after the first threshold in Fig. 7 (d)), the two PL peaks 
Table III. Phase transitions at various temperatures.

\begin{tabular}{ccc}
\hline Temperature $(\mathrm{K})$ & First threshold & Second threshold \\
\hline 8 & polariton condensation & \\
50 & polariton condensation & \\
70 & polariton condensation & photon lasing \\
100 & photon lasing? & \\
200 & photon lasing & \\
\hline
\end{tabular}

are observed in Fig. 6. This fact cannot be explained in the standard photon lasing. Therefore, we cannot claim that this threshold is identified to the transition to the standard photon lasing.

At $8 \mathrm{~K}$, we observe the blue shift behavior after the first threshold [Fig. 5 (a)]. This can be explained as follows: This condensation is taken as the exciton-polariton BEC, which is the weakly-interacting BEC. However, increasing the excitation power, we still observe the PL characteristics similar to those above the first threshold while the system reaches to the nonequilibrium situation. We report that the second threshold is not observed. In the following subsection, we discuss the evaluation of the excitation density of the e-h pair. At $50 \mathrm{~K}$, we also observe the PL characteristics similar to those observed at $8 \mathrm{~K}$ after the first threshold in the blue shift [Fig. 5 (b)], the increasing linewidth [Fig. 7 (b)], and the almost constant second-order autocorrelation function (Fig. 8 (b)). Note that, the effect of all PL characteristics at $50 \mathrm{~K}$ is weaker than that in the $8 \mathrm{~K}$ case. However, at $70 \mathrm{~K}$, we observe that the $\mathrm{PL}$ energy rapidly reaches to the cavity energy; however, we did not observe the blue shift behavior after the first threshold [Fig. 5 (c)], which is completely different from those in the 8 and $50 \mathrm{~K}$ cases. Since the PL linewidth does not be narrow after the first threshold as in 100 and 200 $\mathrm{K}$ cases, this transition cannot correspond to the standard photon lasing. Therefore, this observation may be due to the dynamical condensation of the exciton-polariton system. Thereafter, the same PL characteristics as those in the standard photon lasing is measured after the second threshold.

The above discussions are summarized in Table III to relate our experimental data with our previous knowledge of the exciton-polariton system. While we have not directly measured the $\mathrm{e}-\mathrm{h}$ pair or the $\mathrm{e}-\mathrm{h}$ correlations as alluded previously, we can evaluate the excitation density of the $\mathrm{e}-\mathrm{h}$ pair. Note that the estimated excitation density of the $\mathrm{e}-\mathrm{h}$ pair is not always the same as the actual density of the $\mathrm{e}-\mathrm{h}$ pair since the $\mathrm{e}-\mathrm{h}$ pair 
may break.

\subsection{Estimation of excitation density of the exciton-hole pair}

To determine whether our system reaches the semiconductor inversion condition, i.e., the Bernard-Duraffourg condition, ${ }^{16}$ we evaluated the excitation density using a picosecond mode-locked laser with a $76 \mathrm{MHz}$ repetition rate. By estimating the bandwidth as well as the reflection dip at the energy of the pump laser, we could estimate the input rate as $\sim 0.1$. Considering the absorption coefficient of the QWs at the pumping energy $\left(\sim 2 \times 10^{4} \mathrm{~cm}^{-1}\right)$, the absorption rate at each QW is around 0.014. The absorption probability from an input of one pump photon is $1.4 \times 10^{-3}$, and there are around $2.5 \times 10^{10}$ photons per pulse at an average power of $500 \mathrm{~mW}$. Thus, the excitation density of a spot roughly $50 \times 100 \mu \mathrm{m}$ in size is $1.4 \times 10^{-3} \times 2.5 \times 10^{10} /\left(50 \times 100 \mu \mathrm{m}^{2}\right)=7 \times 10^{11}$ $\mathrm{cm}^{-2}$, which is close to that derived for the pulsed condition given in ref. 16. In ref. 16, the condition was estimated to be close to the Bernard-Duraffourg condition, but the estimated excitation density had a large uncertainty owing to the experimental conditions used. For example, the input rate of 0.1 was estimated roughly and not determined precisely from measurements, as was the absorption rate. Moreover, temperature determination is always a problem in this field. However, the possibility of the system being close to the inversion condition is implicitly implied in the high-excitation regime, and if the inversion condition is satisfied, then this leads to the new type of lasing discussed in the high excitation regime and theoretically studied in the nonequilibrium condition $^{22,23}$ using the framework developed in ref. 33. Although we have not obtained direct evidence of the $\mathrm{e}-\mathrm{h}$ pair correlation in the high-excitation regime, recent observations of a ghost polariton branch ${ }^{34,35}$ using an intense resonant excitation laser indicate the possibility of $\mathrm{e}-\mathrm{h}$ pairs and polaritons even in the high-density regime.

\section{Conclusion}

We observe the intensity, the energy of the main peak, the linewidth, and the secondorder autocorrelation function of the PL from an exciton-polariton system at various temperatures and excitation powers. Our observation is summarized in Table III from the viewpoint of the nonlinear gains of the PL intensity. Contrary to conventional expectations, we did not observe the second threshold at a low-temperature, highexcitation-density regime. The characteristics of the low temperature data differ from those of conventional photon lasing observed at high temperatures. Since we have eval- 
uated the excitation density of $\mathrm{e}-\mathrm{h}$ pairs in an experimental setup that satisfies the Bernard-Duraffourg condition at low temperatures, that is, the high-excitation-density regime, the lasing mechanism of the exciton-polariton system is different from that of a standard photon lasing system. Our results suggest that $\mathrm{e}-\mathrm{h}$ binding is present in the high-excitation regime at low temperatures, supporting the theoretical considerations. ${ }^{19-23}$

\section{Acknowledgments}

The authors wish to thank T. Ogawa, T. Byrnes, K. Kamide, M. Yamaguchi, and N. Ishida for their helpful comments. This research was supported by the Japan Society for the Promotion of Science (JSPS) through its FIRST Program and KAKENHI Grant Numbers 24740277 and 25800181, a Space and Naval Warfare Systems (SPAWAR) Grant N66001-09-1-2024, the Ministry of Education, Culture, Sports, Science and Technology (MEXT), the State of Bavaria, the National Institute of Information and Communications Technology (NICT), and the joint studies program at the Institute for Molecular Science.

\section{Appendix A: Parameters for the Estimation of Effective Masses}

The effective mass of the LP is described by $1 / m_{L P}=|X|^{2} / m_{\text {exc }}+|C|^{2} / m_{\text {cav }}$, where $|X|^{2}$ and $|C|^{2}$ are the Hopfield coefficients determined by the detuning and normalmode splitting of $2 \hbar \Omega$, respectively. Here, the effective mass of the exciton, $m_{\text {exc }}$, is defined by $1 / m_{\text {exc }}=1 / m_{e}+1 / m_{h h}$ in terms of the electron mass $m_{e}$, the heavy hole (hh) mass $m_{h h}$, and the effective mass of the cavity photon $m_{c a v}$. From the dispersion relations, the effective mass $m^{*}$ is calculated as $1 / m^{*}=\left(1 / \hbar^{2}\right) d^{2} E(k) / d k^{2}$. In the range of $k_{\|} \ll k_{\perp}$, the energy of the cavity mass $E_{c a v}=\hbar c / n_{c}\left(k_{\|}^{2}+k_{\perp}{ }^{2}\right)^{1 / 2}$ is approximated by a quadratic curve, and thus, the effective mass at $k_{\|}=0$ is $m_{c a v}=2 \pi \hbar n_{c}{ }^{2} /\left(\lambda_{c} c\right)$ for the refractive index $n_{c}$ and the resonant frequency $\lambda_{c}$. The effective mass at the bottom of the LP branch is $7.46 \times 10^{-35} \mathrm{~kg}$. The following parameters were used to estimate the effective masses: $2 \hbar \Omega=14 \mathrm{meV}$, a free electron mass of $m_{0}=9.109 \times 10^{-31} \mathrm{~kg}$, $m_{e}=0.0665 m_{0}, m_{h h}=0.111 m_{e}, \lambda_{c}=767.5 \mathrm{~nm}, n_{c}=3.6$ for GaAs, and $\Delta E=0$. The fitting region is shown in Fig. A.1.

\section{Appendix B: Effects of Time Integration on the Linewidth Measurements}

At a low temperature $(8 \mathrm{~K})$, time-resolved linewidth data were also collected using a streak camera (Fig. B-1). In contrast to the time-integrated data, the time-resolved 


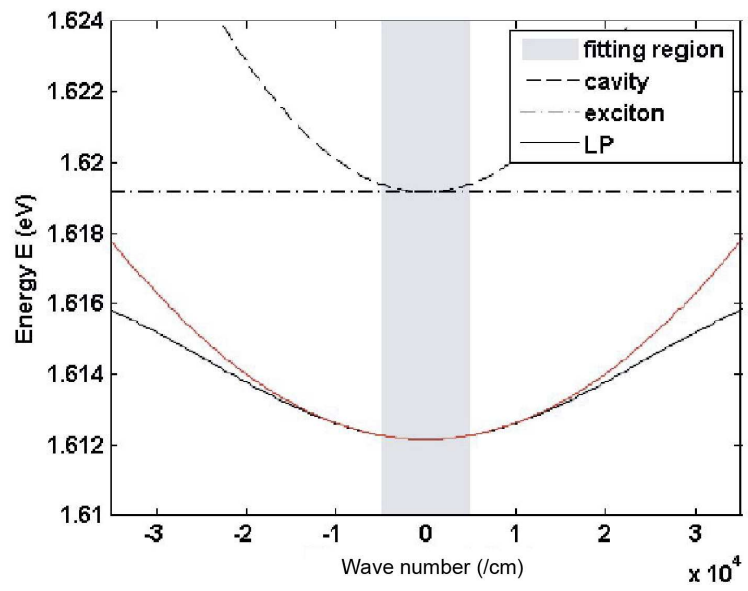

Fig. A.1. (Color Online) Evaluation of the accuracy of the fitting of $E_{L P}\left(k_{\|}\right)=1 / 2\left[E_{\text {exc }}\left(k_{\|}\right)+\right.$ $\left.E_{c a v}\left(k_{\|}\right)-\sqrt{4 \hbar^{2} \Omega^{2}+\left(E_{\text {exc }}\left(k_{\|}\right)-E_{c a v}\left(k_{\|}\right)\right)^{2}}\right]$ (solid black curve) by a quadratic curve (red curve). The fit used 29 data points in the region $\left|k_{\|}\right|<0.5 \times 10^{4} \mathrm{~cm}^{-1}$. The effective mass obtained from the fit was $7.48 \times 10^{-35} \mathrm{~kg}$ with a relative error of $0.26 \%$. From this evaluation, experimental data points in the region $\left|k_{\|}\right|<0.5 \times 10^{4} \mathrm{~cm}^{-1}$ were analyzed to evaluate the effective mass.

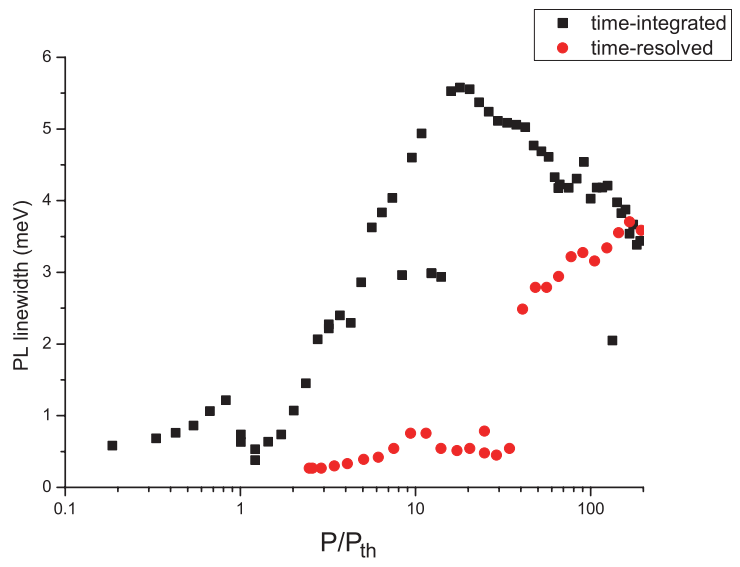

Fig. B•1. (Color Online) Excitation power dependence of the PL linewidth at $8 \mathrm{~K}$. Time-integrated spectroscopy data are shown by black squares, while time-resolved spectroscopy data are shown by red circles. The time-resolved data are extracted at the time corresponding to that of the maximum PL intensity.

data showed a narrower linewidth, especially in the relatively low excitation regime. These data reflect the fact that the degree of blue shift depends on the excitation density and the profile of the pulse pump laser. When the time-varying excitation density was summed, the observed linewidth became broader owing to the sum over the various densities. However, as the excitation increased, the time-resolved data also showed 
a broad linewidth at the time of maximum PL intensity. In the maximum excitation regime, the linewidth was about the same as that for the time-integrated data, which demonstrates that the linewidth was much broader than those in the cases of higher temperatures $(70,100$, and $200 \mathrm{~K})$ corresponding to the highest excitation regimes. The time resolution of streak camera spectroscopy was better than 10 ps owing to the temporal dispersion imposed at the grating of the monochromator attached to the streak camera. ${ }^{16}$ Note also that spatial inhomogeneities can also increase the linewidth, although this effect would appear to be small since we collected the emission near $k=0$ where the intense emission is dominated by the central area of the pump spot. Nevertheless, the difference between the time-integrated and time-resolved measurements in Fig. B 1 reveals that the polariton linewidth of the time-integrated data broadens the obtained results. We also see that this broad linewidth in the relatively low excitation regime is due to time integration rather than to polariton-polariton interactions. However, in the high-excitation regime, it is possible that the broad linewidth is a result of polariton-polariton interactions. 


\section{References}

1) D. Snoke and P. B. Littlewood: Phys. Today 63 (2010) 42.

2) H. Deng, H. Haug, and Y. Yamamoto: Rev. Mod. Phys. 82 (2010) 1489.

3) P. Schwendimann and A. Quattropani: Phys. Rev. B 77 (2008) 085317.

4) H. Deng, G. Weihs, C. Santori, J. Bloch, and Y. Yamamoto: Science 298 (2002) 199.

5) J. Kasprzak, M. Richard, S. Kundermann, A. Baas, P. Jeambrun, J. M. J. Keeling, F. M. Marchetti, M. H. Szyman, R. André, J. L. Staehli, V. Savona, P. B. Littlewood, B. Deveaud, and L. S. Dang: Nature 443 (2006) 409.

6) A. Amo, D. Sanvitto, F. P. Laussy, D. Ballarini, E. del Valle, M. D. Martin, A. Lemaître, J. Bloch, D. N. Krizhanovskii, M. S. Skolnick, C. Tejedor, and L. Viña: Nature 457 (2009) 291.

7) K. G. Lagoudakis, M. Wouters, M. Richard, A. Baas, I. Carusotto, R. André, L. S. Dang, and B. Deveaud-Plédran: Nat. Phys. 4 (2008) 706.

8) G. Roumpos, M. D. Fraser, A. Löffler, S. Höfling, A. Forchel, and Y. Yamamoto: Nat. Phys. 7 (2011) 129.

9) S. Utsunomiya, L. Tian, G. Roumpos, C. W. Lai, N. Kumada, T. Fujisawa, M. Kuwata-Gonokami, A. Löffler, S. Höfling, A. Forchel, and Y. Yamamoto: Nat. Phys. 4 (2008) 700.

10) A. Imamoglu, R. J. Ram, S. Pau, and Y. Yamamoto: Phys. Rev. A 53 (1996) 4250.

11) L. S. Dang, D. Heger, R. André, F. Bøuf, and R. Romenstein: Phys. Rev. Lett. 81 (1998) 3920 .

12) H. Deng, G. Weihs, D. Snoke, J. Bloch, and Y. Yamamoto: Proc. Natl. Acad. Sci. USA 100 (2003) 15318.

13) D. Bajoni, P. Senellart, A. Lemaître, and J. Bloch: Phys. Rev. B 76 (2007) $201305(\mathrm{R})$.

14) B. Nelsen, R. Balili, D. Snoke, L. Pfeiffer, and K. West: J. Appl. Phys. 105 (2009) 122414 .

15) D. Bajoni, P. Senellart, E. Wertz, I. Sagnes, A. Miard, A. Lemaître, and J. Bloch: Phys. Rev. Lett. 100 (2008) 047401. 
16) E. Kammann, H. Ohadi, M. Maragkou, A. V. Kavokin, and P. G. Lagoudakis: New J. Phys. 14 (2012) 105003.

17) J. S. Tempel, F. Veit, M. Assmann, L. E. Kreilkamp, A. Rahimi-Iman, A. Löffler, S. Höfling, S. Reitzenstein, L. Worschech, A. Forchel, and M. Bayer: Phys. Rev. B 85 (2012) 075318.

18) J. S. Tempel, F. Veit, M. Assmann, L. E. Kreilkamp, S. Höfling, M. Kamp, A. Forchel, and M. Bayer: New J. Phys. 14 (2012) 083014.

19) K. Kamide and T. Ogawa: Phys. Rev. Lett. 105 (2010) 056401.

20) T. Byrnes, T. Horikiri, N. Ishida, and Y. Yamamoto: Phys. Rev. Lett. 105 (2010) 186402.

21) K. Kamide and T. Ogawa: Phys. Rev. B 83 (2011) 165319.

22) M. Yamaguchi, K. Kamide, T. Ogawa, and Y. Yamamoto: New J. Phys. 14 (2012) 065001.

23) M. Yamaguchi, K. Kamide, R. Nii, T. Ogawa, and Y. Yamamoto: arXiv:1301.4838 to be published from Phys. Rev. Lett. (2013).

24) S. M. Ulrich, C. Gies, S. Ates, J. Wiersig, S. Reitzenstein, C. Hofmann, A. Löffler, A. Forchel, F. Jahnke, and P. Michler: Phys. Rev. Lett. 98 (2007) 043906.

25) J. Kasprzak, M. Richard, A. Baas, B. Deveaud, R. André, J.-Ph. Poizat, and L. S. Dang: Phys. Rev. Lett. 100 (2008) 067402.

26) D. Sarchi, P. Schwendimann, and A. Quattropani: Phys. Rev. B 78 (2008) 073404.

27) T. D. Doan, H. Thien Cao, D. B. Tran Thoai, and H. Haug: Phys. Rev. B 78 (2008) 205306.

28) M. Wouters and V. Savona: Phys. Rev. B 79 (2009) 165302.

29) H. Haug, T. D. Doan, H. Thien Cao, and D. B. Tran Thoai: Phys. Rev. B 85 (2012) 205310.

30) T. Horikiri, P. Schwendimann, A. Quattropani, A. Löffler, S. Höfling, A. Forchel, and Y. Yamamoto: Phys. Rev. B 81 (2010) 033307.

31) D. Porras and C. Tejedor: Phys. Rev. B 67 (2003) 161310(R).

32) P. Tsotsis, P. S. Eldridge, T. Gao, S. I. Tsinztos, Z. Hatzopoulos, and P. G. Savvidis: New J. Phys. 14 (2012) 023060. 
33) M. H. Szymańska, J. Keeling, and P. B. Littlewood: Phys. Rev. Lett. 96 (2006) 230602.

34) V. Kohnle, Y. Léger, M. Wouters, M. Richard, M. T. Portella-Oberli, and B. Deveaud-Plédran: Phys. Rev. Lett. 106 (2011) 255302.

35) J. M. Zajac and W. Langbein: arXiv:1210.1455 (2012). 